Journal of Antimicrobial Chemotherapy (1990) 26, Suppl. B, 7-15

\title{
Usual and unusual antibacterial effects of quinolones
}

\author{
Y. X. Furet and J.-C. Pechère
}

\author{
Department of Microbiology, CMU, 1211 Geneva 4, Switzerland
}

\begin{abstract}
Recently documented antibacterial effects of quinolones are reviewed. DNA gyrase is most likely to be the primary target site for these agents. Quinolones rapidly kill susceptible bacteria; the mechanisms of the bactericidal activity, still poorly understood, probably involve new protein synthesis. Quinolones alter membrane integrity before cell death, leading to leakage of cytoplasmic constituents. In Gram-negative bacteria, quinolones act as chelating agents for outer membrane divalent cations, disorganizing the bacterial lipopolysaccharide layer and facilitating the further entry of quinolone molecules in a 'self-promoted' pathway. Quinolones inhibit plasmid replication and reduce the efficacy of plasmid conjugation. Subinhibitory concentrations of quinolones can interfere with bacterial virulence factors, such as bacterial adherence to the host cell, phagocytosis and production of enzymes implicated in virulence. Recent studies also indicate synergism of quinolones with oxacilin against methicillin-resistant staphylococci and describe improved activity of newer compounds against Gram-positive pathogens.
\end{abstract}

\section{Introduction}

Development of quinolone agents has been impressive during recent years, accounting for their wide use in clinical practice. These drugs have shown a remarkable potency against usual pathogens, as well as against bacteria that are less common and difficult to treat, including multiply resistant nosocomial bacteria and various intracellular pathogens. How quinolones act against these numerous organisms, however, is not completely understood. This review will focus on some recently discovered antibacterial effects of quinolones.

\section{Quinolones inhibit DNA gyrase functions}

DNA gyrase is an enzyme capable of introducing negative supercoils into bacterial DNA, and responsible for the accommodation of the long circular chromosome within the limited space provided by the cell envelope. The principal prerequisite for the various and complex activities of the enzyme is its ability to cleave and rejoin doublestranded DNA.

Most of the multiple biochemical functions of DNA gyrase on cell physiology are affected as a result of the quinolone activity, including the inhibition of DNA replication, post-replicational repair (Priel, 1984), recombination of DNA and separation of the two circular daughter chromosomes in the terminal phase of replication (Smith, 1986; Sutcliffe, Gootz \& Barrett, 1989). Yet exactly how quinolones interfere with DNA gyrase function remains unclear or controversial. Do quinolones exert their effect by binding to DNA gyrase? Such a hypothesis can be supported by several observations: inhibition of the enzymatic activity of purified DNA gyrase by quinolones 
(Barrett, Sutcliffe \& Gootz, 1990), bacterial resistance associated with DNA gyrase mutations (Gellert et al., 1977; Sato et al., 1986) and ${ }^{19} \mathrm{~F}$ nuclear resonance experiments showing the affinity of pefloxacin for DNA gyrase (Le Goffic, 1985). However, if quinolones bind directly to DNA gyrase, the exact binding site remains to be determined. Other reports have suggested that quinolones bind to DNA, and not directly to DNA gyrase (Shen \& Pernet, 1985; Tornatelli \& Pedrini, 1988) or to the tertiary DNA gyrase-DNA complex (Shen et al., 1989). As a final remark, a computer program, matching experimental data pertaining to DNA gyrase inhibition with quinolone structures predicted with acceptable accuracy the consequences of structural variations for MICs (Klopman et al., 1987): DNA gyrase is indeed a primary target for the quinolones.

\section{Killing of bacteria by quinolones: a puzzling question}

Since DNA gyrase inhibition blocks bacterial replication, the expected effect of quinolones would be bacteriostatic. In fact, exposure of a susceptible bacterial population to a quinolone results generally in rapid cell death, followed by a plateau at a viable cell count about $10^{-4}$-fold below the initial inoculum (Wolfson, Hooper \& Swartz, 1989). Bactericidal activity can even be shown during the stationary phase of growth (Zeiler, 1985).

The mechanisms of these lethal events remain unclear. Killing is reduced, paradoxically, by increasing the quinolone concentration over a certain level and is also markedly inhibited by adding rifampicin or chloramphenicol (Crumplin, Kenwright \& Hirst, 1984). Rifampicin, chloramphenicol and high quinolone concentrations have in common the capacity to inhibit protein synthesis suggesting that the killing activity by quinolones is associated with new protein synthesis.

\section{Quinolones and SOS response}

One possible explanation for the bactericidal activity is that quinolones cause DNA gyrase to damage DNA (Smith, 1984), inducing the SOS DNA repair system. Celldamaging environmental conditions trigger the synthesis of specific cascades of proteins, whose physiological role is to repair the affected cellular system, and protect it from further damage. Among other phenomena, this SOS response enhances DNA repair capacity and mutagenesis and blocks cell division (producing filamentation) and cell respiration (Little \& Mount, 1982). The quinolones were found to trigger the SOS response, with a maximum intensity at the quinolone concentration that produced the greatest bactericidal effect (Phillips et al., 1987). In SOS mutants however, quinolone killing did not correlate necessarily with the SOS phenotype; furthermore induction of the SOS response was protective to fleroxacin and enoxacin treated bacteria, but not to ciprofloxacin treated bacteria (Walters, Piddock \& Wise, 1989). Taken in aggregate, these experiments dealing with the SOS response do not close the discussions about the killing, but suggest that the mechanisms of action of different quinolones are not uniform in their precise detail, as was originally thought.

\section{Loss of membrane integrity}

Electron microscope studies showed that quinolone treated Escherichia coli cells displayed areas of reduced cytoplasmic contrast at the time when cell death occurred 
(Dougherty \& Saukkonen, 1985). At the same time, the cells became more susceptible to detergent, and exhibited increased membrane permeability. These quinolone-induced changes were attributed to the loss of membrane integrity, leading to leakage of cytoplasmic constituents, and, ultimately, cell death (Dougherty \& Saukkonen, 1985). Again in Esch. coli, quinolones induced lipopolysaccharide release and increased cell surface hydrophobicity (Chapman \& Georgopapadakou, 1988). The same authors showed evidence that quinolones acted as chelating agents vis-à-vis outer membranebound divalent cations, notably $\mathbf{M g}^{2+}$ which is well known to antagonize quinolone antibacterial activity (Auckenthaler, Michéa-Hamzehpour \& Pechère, 1986). This chelating effect leads to disorganization of this bacterial surface layer, facilitating the further entry of quinolone molecules through the outer membrane in a 'self-promoted' pathway similar to that described for the aminoglycosides (Hancock, Raffle \& Nicas, 1981). An alternative route for penetration through the outer membrane is through the water filled protein porin channels. It is likely that for Esch.coli quinolone molecules may enter either way, the primary route depending on their solubility: the more hydrophilic compounds via the porin channels and hydrophobic compounds via the 'self-promoted' pathway.

\section{Paradoxical quinolone action on phage replication}

Additional insight into the mode of action of quinolones is given by experiments performed with phage T7 which does not seem to require DNA gyrase for growth in Esch. coli, since a normal phage burst size occurs at an elevated temperature in host bacteria containing a thermosensitive DNA gyrase. Strikingly, virus replication leading to the same burst was preserved in the presence of nalidixic acid at the elevated, but not at the permissive temperature. Hence inhibition of phage $T 7$ was thought to be due to formation of an irreversible quinolone-gyrase complex, which did not simply have a passive role in blocking a cascade of biochemical reactions, but was thought to act as a poison, mediating cell death (Kreuzer \& Cozzarelli, 1979).

\section{Filamentation}

Filamentation, caused by the inhibition of cell division, occurs before cell death and may result, at least in part, from antagonism of DNA synthesis and induction of the SOS response (Phillips et al., 1987). Filamentation might also be involved in the killing action of quinolones, since a mutant of Esch. coli, incapable of filamenting, appeared to be less susceptible to quinolones than was the parent strain (Walters et al., 1989). Cell enlargement and cessation of septation were also observed in Staphylococcus aureus after norfloxacin treatment in a fashion very similar to the alterations produced by cephalexin, which binds almost exclusively to PBP3 (Georgopapadakou, Dix \& Mauriz, 1986). Since a significant increase of PBP synthesis has been shown in filaments obtained from nalidixic acid-treated Esch. coli cells (Amaral, Schwarz \& Lorian, 1986), a direct interaction between quinolone agents and PBPs (or PBP metabolism) represents a hypothesis deserving further investigation.

\section{Unexpected oxacillin-quinolone synergism against methicillin-resistant staphylococei}

According to fractional inhibitory concentration indices and killing kinetics, the combination of a quinolone with oxacillin was regularly synergistic or additive against 
methicillin-resistant staphylococci (Rohner et al., 1989). Little or no interaction occurred when the same combinations were tested against methicillin-susceptible strains. At first sight, the synergism was somewhat surprising, since the target of oxacillin, that is the bacterial cell wall, appears quite independent from the target of quinolones. Explanatory hypotheses include direct or indirect interactions of quinolones with the PBPs (especially PBP2a which accounts for methicillin resistance) and quinolone induced plasmid cures, since PBP2a regulator genes are known to be located on plasmids. Even though they are little understood, these observations provide invitro evidence for the potential clinical use of quinolones in combination with a $\beta$-lactamase resistant penicillin in treating infections due to methicillin-resistant staphylococci.

\section{Quinolones and plasmids}

In vitro, quinolones were found to eliminate plasmids of Esch. coli at subinhibitory concentrations (Weisser \& Wiedemann, 1986). This effect resulted from inhibition of plasmid replication, which seemed to be more sensitive to the quinolone action than chromosomal replication. Curing of plasmids by quinolones remains incomplete and limited however; it is unlikely to be of clinical relevance in the individual patient, but may have ecological implications when quinolones are widely used (Platt \& Black, 1987). Furthermore, an Esch. coli mating system showed that various quinolones were able to reduce by $90 \%$ the efficiency of plasmid conjugation when concentrations were one to six times the MIC for the parent strain (Weisser \& Wiedemann, 1987). Again, the phenomenon is probably not clinically important, since the lethal effect of the drug was predominant over the potential inhibition of plasmid transfer. Certainly more pertinent for the clinician is the remarkable scarcity of plasmid-mediated quinolone resistance. As a rare example, a transferable resistance to nalidixic acid has been shown in Shigella dysenteriae and Esch. coli (Munshi et al., 1987).

\section{Subinhibitory concentrations of quinolones can alter bacterial virulence}

The strength of the attractive and repulsive forces involved in bacterial surface interactions with nearby cells are of prime importance for bacterial attachment to host cells, which is a key step in bacterial pathogenesis. Antibiotics that alter the bacterial surface can alter bacterial adhesion (Schifferli \& Beachey, 1988) and subsequently phagocytosis. Quinolones do produce bacterial surface changes and, not surprisingly, interfere with the adherence process. Subinhibitory concentrations of pefloxacin ( $\frac{1}{2}$ to $\frac{1}{8}$ the MIC) markedly diminished the capacity of Staph. aureus for adhesion to buccal cells (Desnottes et al., 1987). Whether these effects involve DNA gyrase or represent another drug action remains as yet unclear, but they may have a clinical impact. In a dog model of arterial grafts, subinhibitory concentrations of pefloxacin were efficient in protecting the foreign body material from Staph. aureus infection after bacteraemic challenge (J.-C. Pechère, unpublished observation). However, sub-MICs of quinolones (pefloxacin was not investigated) had no effect on the adherence of slime-producing Staph. epidermidis to vascular prostheses in an in-vitro system (Schmitt et al., 1989), a discrepancy probably due to the absence of host factors in the latter experiments. Other investigations showed that subinhibitory concentrations of pefloxacin decreased the adhesion of Esch. coli to human uroepithelial cells, and that this effect was associated with a decrease in P-fimbria formation (Desnottes, Le Roy \& Diallo, 1988). In good 
agreement with this, quinolones were also capable of altering the affinity of various Enterobacteriaceae for the ileal mucosal cell surface in vitro (Edmiston \& Goheen, 1989). Subinhibitory concentrations of ciprofloxacin also reduced Pseudomonas aeruginosa tissue injury in a rat lung model. This effect was attributed to a decrease in elastase and exoenzyme $S$ production in one non-mucoid isolate, but remained unexplained in a mucoid isolate (Grimwood et al., 1989).

\section{The post-antibiotic effect (PAE)}

PAE refers to a temporally limited suppression of regrowth of organisms that have been exposed to an antimicrobial agent for a certain period.

Quinolones have been shown to produce a prolonged ( $4 \mathrm{~h}$ or more) PAE, in both Gram-positive and Gram-negative isolates (Neu et al., 1987). Of particular interest, it seems that the effect can be demonstrated after exposure to quinolones at an acidic $\mathrm{pH}$ as well as at high $\mathbf{M g}^{2+}$ concentrations, that is, in conditions that are unfavourable to quinolone antibacterial action. Organisms resistant to quinolones also showed PAE after treatment with quinolone concentrations that are achievable in urine (Neu et al., 1987).

\section{Recently documented antibacterial activity of quinolones}

The wide antibacterial spectrum of the new generation of quinolones is now well established (Bellido \& Pechère, 1989; Wolfson \& Hooper, 1989), but some of the recently documented antibacterial activities may broaden present clinical applications. Several newer compounds have shown enhanced potency against Gram-positive pathogens: temafloxacin (Nye et al., 1989), sparfloxacin (Kojima, Inoue \& Mitsuhashi, 1989), WIN 57273 (Jones \& Barry, 1990) and Am-1091 (Neu, Novelli \& Chin, 1989), to quote only a few. These improvements would be of great interest since infections with Streptococcus pneumoniae, Listeria monocytogenes and even Enterococcus faecalis, which have hardly been amenable to quinolone therapy until now, would be in reach in the future. This, however, will require clinical confirmation. The genus Nocardia is another example of a pathogen which shows limited susceptibility to the presently marketed quinolones but is more effectively inhibited by a newer compound, tosufloxacin (Yazawa, Mikami \& Uno, 1989).

Several recent studies (Leysen, Haemers \& Pattyn, 1989) have confirmed a promising antimycobacterial activity. Quinolones penetrate readily into host cells, a relevant property for treating tuberculosis and leprosy. Hence selected quinolone agents are likely to increase our therapeutic weaponry against these infections. Of the commercially available agents, ofloxacin and ciprofloxacin appear most active, but investigational drugs such as sparfloxacin, and WIN 57273. showed even greater potency. Susceptible mycobacterial species include Mycobacterium tuberculosis (Fenlon \& Cynamon, 1986; Gorzynski, Gutman \& Allan, 1989), M.paratuberculosis (Chiodini, 1990), M. leprae (Franzblau, 1989; Ramasesh, Krahenbuhl \& Hastings, 1989; Franzblau \& White, 1990), M. avium complex (Khardori et al., 1989) and rapidly growing mycobacterial species (Wallace et al., 1990). In-vitro synergistic activity between ethambutol and fluorinated quinolones was observed against $M$. avium complex, probably associated with an enhanced penetration of the quinolone induced by ethambutol (Hoffner et al., 1989). Clinical experience is as yet limited. Ofloxacin (Tsukamura, Mizuno \& Toyama, 1986) and pefloxacin (Fu et al., 1987) have been used 
with success in combined therapy for treating tuberculosis. Ofloxacin resistance emerged in cases in which ofloxacin was the only active drug administered (Tsukamura et al., 1985). Other intracellular pathogens against which quinolones have gained recent attention are the Legionella species, which showed a good response in an animal model (Saito et al., 1986), and Coxiella burnetii (Raoult, Yeaman \& Baca, 1989; Yeaman, Roman \& Baca, 1989) which was very susceptible to ofloxacin and pefloxacin.

\section{Conclusions}

The first fluoroquinolone, pefloxacin, was developed less than ten years ago. During the last decade we have witnessed a rapid increase in information regarding this class of antimicrobial agents, due to the development of new compounds, broadening clinical indications, and increasing knowledge of molecular aspects. Obviously, the field remains very active, and we shall have to follow developments in the years to come.

\section{Acknowledgements}

This work was supported by the Fonds National Suisse de la Recherche Scientifique (Grant 31-28007.89).

\section{References}

Amaral, L., Schwarz, U. \& Lorian, V. (1986). Penicillin-binding proteins of filaments of Escherichia coli induced by low concentrations of nalidixic acid, oxolinic acid, novobiocin or nitrofurantoin. Drugs Under Experimental and Clinical Research 12, 653-6.

Auckenthaler, R., Michéa-Hamzehpour, M. \& Pechère, J. C. (1986). In-vitro activity of newer quinolones against aerobic bacteria. Journal of Antimicrobial Chemotherapy 17, Suppl. B, 29-39.

Barrett, J. F., Sutcliffe, J. A. \& Gootz, T. D. (1990). In-vitro assays used to measure the activity of topoisomerases. Antimicrobial Agents and Chemotherapy 34, 1-7.

Bellido, F. \& Pechère, J. C. (1989). Laboratory survey of fluoroquinolone activity. Reviews of Infectious Diseases 11, S917-24.

Chapman, J. S. \& Georgopapadakou, N. H. (1988). Routes of quinolone permeation in Escherichia coli. Antimicrobial Agents and Chemotherapy 32, 438-42.

Chiodini, R. J. (1990). Bactericidal activities of various antimicrobial agents against human and animal isolates of Mycobacterium paratuberculosis. Antimicrobial Agents and Chemotherapy 34, 366-7.

Crumplin, G. C., Kenwright, M. \& Hirst, T. (1984). Investigations into the mechanism of action of the antibacterial agent norfloxacin. Journal of Antimicrobial Chemotherapy 13, Suppl. B, 9-23.

Desnottes, J. F., Diallo, N., Moret, G. \& Santonja, R. (1987). Effects of subinhibitory concentrations of pefloxacin on the adherence of Staphylococcus aureus to human cells. Drugs Under Experimental and Clinical Research 13, 69-73.

Desnottes, J. F., Le Roy, D. \& Diallo, N. (1988). Effect of subminimal inhibitory concentrations of pefloxacin on the piliation and adherence of E. coli. Drugs Under Experimental and Clinical Research 14, 629-34.

Dougherty, T. J. \& Saukkonen, J. J. (1985). Membrane permeability changes associated with DNA gyrase inhibitors in Escherichi coli. Antimicrobial Agents and Chemotherapy 28, 200-6.

Edmiston, C. E. \& Goheen M. P. (1989). Impact of subinhibitory concentrations of quinolones on adherence of Enterobacteriaceae to cells of the small bowel. Reviews of Infectious Diseases 11, Suppl. 5, S948-9.

Fenlon, C. H. \& Cynamon, M. H. (1986). Comparative in vitro activities of ciprofloxacin and other 4-quinolones against Mycobacterium tuberculosis and Mycobacterium intracellulare. Antimicrobial Agents and Chemotherapy 29, 386-8. 
Franzblau S. G. (1989). Drug susceptibility testing of Mycobacterium leprae in the BACTEC 460 system. Antimicrobial Agents and Chemotherapy 33, 2115-7.

Franzblau, S. G. \& White, K. E. (1989). Comparative in vitro activities of 20 fluroquinolones against Mycobacterium leprae. Antimicrobial Agents and Chemotherapy 34, 229-31.

Fu, K. P., Hetzel, N., Gregory, F. J. \& Hung, P. P. (1987). Therapeutic efficacy of cefpiramideciprofloxacin combination in experimental pseudomonas infections in neutropenic mice. Journal of Antimicrobial Chemotherapy 20, 541-6.

Gellert, M., Mizuuchi, K., O'Dea, M. H., Itoh, T. \& Tomizawa J.-I. (1977). Nalidixic acid resistance: a second genetic character involved in DNA gyrase activity. Proceedings of the National Academy of Sciences USA 74, 4772-6.

Georgopapadakou, N. H., Dix, B. A. \& Mauriz, Y. R. (1986). Possible physiological functions of penicillin-binding proteins in Staphylococcus oureus. Antimicrobial Agents and Chemotherapy 29, 333-6.

Gorzynski, E. A., Gutman, S. I. \& Allen, W. (1989). Comparative antimycobacterial activities of difloxacin, temafloxacin, enoxacin, pefloxacin, reference fluoroquinolones, and a new macrolide, clarithromycin. Antimicrobial Agents and Chemotherapy 33, 591-2.

Grimwood, K., To, M., Rabin, H. R. \& Woods, D. E. (1989). Subinhibitory antibiotics reduce Pseudomonas aeruginosa tissue injury in the rat lung model. Journal of Antimicrobial Chemotherapy 24, 937-45.

Hancock, R. E., Raffle, V. J. \& Nicas, T. I. (1981). Involvement of the outer membrane in gentamicin and streptomycin uptake and killing in Pseudomonas aeruginosa. Antimicrobial Agents and Chemotherapy 19, 777-85.

Hoffner, S. E., Kratz, M., Olsson-Liljequist, B., Svenson, S. B. \& Källenius, G. (1989). In-vitro synergistic activity between ethambutol and fluorinated quinolones against Mycobacterium avium complex. Journal of Antimicrobial Chemotherapy 24, 317-24.

Jones, R. N. \& Barry, A. L. (1990). In vitro evaluation of WIN 57273, a new broad-spectrum fluoroquinolone. Antimicrobial Agents and Chemotherapy 34, 306-13.

Khardori, N., Rolston, K., Rosenbaum, B., Hayat, S. \& Bodey G. P. (1989). Comparative invitro activity of twenty antimicrobial agents against clinical isolates of Mycabacterium avium complex. Journal of Antimicrobial Chemotherapy 24, 667-73.

Klopman, G., Macina, O. T., Levinson, M. E. \& Rosenkranz, H. S. (1987). Computer automated structure evaluation of quinolone antibacterial agents. Antimicrobial Agents and Chemotherapy 31, 1831-40.

Kojima, T., Inoue, M. \& Mitsuhashi, S. (1989). In vitro activity of AT-4140 against clinical bacterial isolates. Antimicrobial Agents and Chemotherapy 33, 1980-8.

Kreuzer, K. N. \& Cozzarelli, N. R. (1979). Escherichia coli mutants thermosensitive for deoxyribonucleic acid gyrase subunit $A$ : effects on deoxyribonucleic acid replication, transcription, and bacteriophage growth. Journal of Bacteriology 140, 424-35.

Le Goffic, F. (1985). Les quinolones, möchanismes d'action. In Les Nouvelles Quinolones (Pocidalo, J. J., Vachon, F. \& Regnier, B., Eds), pp. 15-23. Edition Arnette, Paris.

Leysen, D. C., Haemers, A. \& Pattyn, S. R. (1989). Mycobacteria and the new quinolones. Antimicrobial Agents and Chemotherapy 33, 1-5.

Little, J. W. \& Mount, D. W. (1982). The SOS regulatory system of Escherichia coli. Cell 29, $11-22$.

Munshi, M. H., Haider, K. Rahaman, M. M., Sack, D. A., Ahmed, Z. U. \& Morshed, M. G. (1987). Plasmid-mediated resistance to nalidixic acid in Shigella dysenteriae type 1. Lancet ii, 419-21.

Neu, H. C., Kumada, T., Chin, N.-X. \& Mandell, W. (1987). The post-antimicrobial suppressive effect of quinolone agents. Drugs under Experimental and Clinical Research 13, 63-7.

Neu, H. C., Novelli, A. \& Chin, N.-X. (1989). Comparative in vitro activity of a new quinolone, AM-1091. Antimicrobial Agents and Chemotherapy 33, 1036-41.

Nye, K., Shi, Y. G., Andrews, J. M., Ashby, J. P. \& Wise, R. (1989). The in-vitro activity, pharmacokinetics and tissue penetration of temafloxacin. Journal of Antimicrobial Chemotherapy 24, 415-24.

Phillips, I., Culebras, E., Moreno, F. \& Baquero, F. (1987). Induction of the SOS response by new 4-quinolones. Journal of Antimicrobial Chemotherapy 20, 631-8.

Platt, D. J. \& Black, A. C. (1987). Plasmid ecology and the elimination of plasmids by 4-quinolones. Journal of Antimicrobial Chemotherapy 20, 137-42. 
Priel, E. (1984). Inhibition of the postreplication recombinational repair by DNA-gyrase antagonists in Escherichia coli. Cell Biology International Reports 8, 773-86.

Ramasesh, N., Krahenbuhl, J. L. \& Hastings, R. C. (1989). In vitro effects of antimicrobial agents on Mycobacterium leprae in mouse peritoneal macrophages. Antimicrobial Agents and Chemotherapy 33, 657-62.

Raoult, D., Yeaman, M. R. \& Baca, O. G. (1989). Susceptibility of Coxiella burnetii to pefloxacin and ofloxacin in ovo and in persistently infected L929 cells. Antimicrobial Agents and Chemotherapy 33, 621-3.

Rohner, P., Herter, C., Auckenthaler, R., Pechère, J.-C., Waldvogel, F. A. \& Lew, D. P. (1989). Synergistic effect of quinolones and oxacillin on methicillin-resistant Staphylococcus species. Antimicrobial Agents and Chemotherapy 33, 2037-41.

Saito, A., Koga, M., Shigeno, H., Watanabe, K., Mori, K., Kohno, S. el al. (1986). The antimicrobial activity of ciprofloxacin against Legionella species and the treatment of experimental Legionella pneumonia in guinea pigs. Journal of Antimicrobial Chemotherapy 18, $251-60$.

Sato, K., Inoue, Y., Fujii, T., Aoyama, H., Inoue, M. \& Mitsuhashi, S. (1986). Purification and properties of DNA gyrase from a fluoroquinolone-resistant strain of Escherichia coli. Antimicrobial Agents and Chemotherapy 30, 777-80.

Schifferli, D. M. \& Beachey, E. H. (1988). Bacterial adhesion: modulation by antibiotics with primary targets other than protein synthesis. Antimicrobial Agents and Chemotherapy 32, 1609-13.

Schmitt, D. D., Bandyk, D. F., Edmiston, C. E., Levy, M. F., Seabrook, G. R. \& Towne, J. B. (1989). The in vitro effect of subinhibitory concentrations of quinolones and vancomycin on adherence of slime-producing Staphylococcus epidermidis to vascular prostheses. Reviews of Infectious Diseases 11, Suppl. 5, S947-8.

Shen, L. L., Kohlbrenner, W. E., Weigl, D. \& Baranowski, J. (1989). Mechanisms of quinolone inhibition of DNA gyrase. Journal of Biological Chemistry 264, 2973-8.

Shen, L. L. \& Pernet, A. G. (1985). Mechanisms of inhibition of DNA gyrase of analogues of nalidixic acid: the target of the drugs is DNA. Proceedings of the National Academy of Sciences USA 82, 307-11.

Smith, J. T. (1984). Awakening the slumbering potential of the 4-quinolone antibacterials. Pharmaceutical Journal 15, 299-305.

Smith, J. T. (1986). The mode of action of 4-quinolones and possible mechanisms of resistance. Journal of Antimicrobial Chemotherapy 18, Suppl. D. 21-9.

Sutcliffe, J. A., Gootz, T. D. \& Barrett, J. F. (1989). Biochemical characteristics and physiological significance of mayor DNA topoisomerases. Antimicrobial Agents and Chemotherapy 33, 2027-33.

Tornatelli, S. \& Pedrini, A. M. (1988). Studies on the interaction of 4-quinolones with DNA by DNA unwinding experiments. Biochimica et Biophysica Acta 949, 279-87.

Tsukamura, M., Mizuno, S. \& Toyama, H. (1986). Comparison of in-vitro growth-inhibitory activities of ofloxacin, ciprofloxacin and norfloxacin against various species of mycobacteria. Kekkaku 61, 453-9.

Tsukamura, M., Nakamura, E., Yoshii, S. \& Amano, H. (1985). Therapeutic effect of a new antibacterial substance ofloxacin (DL-8280) on pulmonary tuberculosis. American Review of Respiratory Disease 131, 352-6.

Wallace, R. J., Bedsole, G., Sumter, G., Sanders, C. V., Steele, L. C., Brown, B. A. et al. (1990). Activities of ciprofloxacin and ofloxacin against rapidly growing mycobacteria with demonstration of acquired resistance following single-drug therapy. Antimicrobial Agents and Chemotherapy 34, 65-70.

Walters, R. N., Piddock, L. J. V. \& Wise, R. (1989). The effects of mutations in the SOS response on the kinetics of quinolone killing. Journal of Antimicrobial Chemotherapy 24, 863-73.

Weisser, J. \& Wiedemann, B. (1986). Elimination of plasmids by enoxacin and ofloxacin at near inhibitory concentrations. Journal of Antimicrobial Chemotherapy 18, 575-83.

Weisser, J. \& Wiedemann, B. (1987). Inhibition of R-plasmid transfer in Escherichia coli by 4-quinolones. Antimicrobial Agents and Chemotherapy 31, 531-4.

Wolfson, J. S. \& Hooper, D. C. (1989). Fluoroquinolone antimicrobial agents. Clinical Microbiology Reviews 2, 378-424. 
Wolfson, J. S., Hooper, D. C. \& Swartz, M. N. (1989). Mechanisms of action of and resistance to quinolone antimicrobial agents. In Quinolone Antimicrobial Agents (Wolfson, J. S. \& Hooper, D. C., Eds), pp. 35-70. American Society for Microbiology, Washington, DC.

Yazawa, K., Mikami, Y. \& Uno J. (1989). In vitro susceptibility of Nocardia spp. to a new fluoroquinolone, tosufloxacin (T-3262). Antimicrobial Agents and Chemotherapy 33, 2140-1.

Yeaman, M. R., Roman, M. J. \& Baca, O. G. (1989). Antibiotic susceptibilities of two Coxiella burnetii isolates implicated in distinct clinical syndromes. Antimicrobial Agents and Chemotherapy 33, 1052-7.

Zeiler, H.-J. (1985). Evaluation of the in vitro bacterial action of ciprofloxacin on cells of Escherichia coli in the logarithmic and stationary phases of growth. Antimicrobial Agents and Chemotherapy 28, 524-7. 